December 2017

\title{
Characterizations of Distributions by Expected Values of Lower Record Statistics with Spacing
}

\author{
M. Faizan \\ Aligarh Muslim University, Aligarh, India, mdfaizan02@gmail.com \\ Ziaul Haque \\ National Sample Survey Office, New Delhi, India, ziaulstats@gmail.com \\ M.A. Ansari \\ Aligarh Muslim University, Aligarh, India
}

Follow this and additional works at: http://digitalcommons.wayne.edu/jmasm

Part of the Applied Statistics Commons, Social and Behavioral Sciences Commons, and the Statistical Theory Commons

\section{Recommended Citation}

Faizan, M., Haque, Z., \& Ansari, M. A. (2017). Characterizations of Distributions by Expected Values of Lower Record Statistics with Spacing. Journal of Modern Applied Statistical Methods, 16(2), 310-321. doi: 10.22237/jmasm/1509495360

This Regular Article is brought to you for free and open access by the Open Access Journals at DigitalCommons@WayneState. It has been accepted for inclusion in Journal of Modern Applied Statistical Methods by an authorized editor of DigitalCommons@WayneState. 


\section{Characterizations of Distributions by Expected Values of Lower Record Statistics with Spacing}

\section{Faizan}

Aligarh Muslim University

Aligarh, India

\author{
Ziaul Haque \\ National Sample Survey Office \\ New Delhi, India
}

\author{
M. A. Ansari \\ Aligarh Muslim University \\ Aligarh, India
}

The characterizations of a certain class of probability distributions are established through conditional expectation of lower record values when the conditioned record value may not be the adjacent one. Some of its important deductions are also discussed.

Keywords: Characterization, continuous distributions, conditional expectation, lower record values

\section{Introduction}

Record values have been extensively studied in literature. For some excellent reviews, see Ahsanullah (1995), Arnold, Balakrishnan, and Nagaraja (1998), and Nevzorov (2001). Characterization of a probability distribution plays an important role in the determination of distributions by using certain characteristics in the given data. Different methods were used to identify several types of distributions. Conditional expectations of record values were extensively used in characterizing the continuous probability distributions. For examples, consider Malinowska and Szynal (2008), Shawki and Bakoban (2009), and, recently, Yanev (2012), Ahsanullah, Shakil, and Golam Kibria (2013), Azedine (2013), and Nadarajah, Teimouri, and Shih (2014), among others.

Let $X_{1}, X_{2}, \ldots$ be a sequence of independent, identically-distributed continuous random variables with distribution function (df) $\mathrm{F}(x)$ and probability density function (pdf) $\mathrm{f}(x)$. Let $X_{\mathrm{L}(r)}$ be the $r^{\text {th }}$ lower record value; then the conditional pdf of $X_{\mathrm{L}(s)}$ given $X_{\mathrm{L}(r)}=x, 1 \leq r<s$, is (Ahsanullah, 1995)

M. Faizan is in the Department of Statistics and Operations Research. Email them at: mdfaizan02@gmail.com. Ziaul Haque is in the Field Operations Division. Email them at: ziaulstats@gmail.com. M. A. Ansari is in the Department of Statistics and Operations Research. 


$$
\mathrm{f}\left(X_{\mathrm{L}(s)} \mid X_{\mathrm{L}(r)}=x\right)=\frac{1}{\Gamma(s-r)}[-\ln \mathrm{F}(y)+\ln \mathrm{F}(x)]^{s-r-1} \frac{\mathrm{f}(y)}{\mathrm{F}(x)}
$$

and the conditional pdf of $X_{\mathrm{L}(r)}$ given $X_{\mathrm{L}(s)}=y, 1 \leq r<s$, is (Ahsanullah, 1995)

$$
\begin{aligned}
& \mathrm{f}\left(X_{\mathrm{L}(r)} \mid X_{\mathrm{L}(s)}=y\right) \\
& \quad=\frac{\Gamma(s)}{\Gamma(r) \Gamma(s-r)} \frac{[-\ln \mathrm{F}(x)]^{r-1}}{[-\ln \mathrm{F}(y)]^{s-1}}[-\ln \mathrm{F}(y)+\ln \mathrm{F}(x)]^{s-r-1} \frac{\mathrm{f}(x)}{\mathrm{F}(x)}
\end{aligned}
$$

Let $X$ be a continuous random variable with df $\mathrm{F}(x)$ defined by:

$$
\begin{gathered}
\mathrm{F}(x)=[a \mathrm{~h}(x)+b]^{c}, x \in(\alpha, \beta) \\
\mathrm{F}(x)=\exp \left[-e^{-a \mathrm{~h}(x)+b}\right], x \in(\alpha, \beta)
\end{gathered}
$$

where $a, b$, and $c$ are constants and $\mathrm{h}(x)$ is a monotonic and differentiable function of $x$ defined on $(\alpha, \beta)$ such that $\mathrm{F}(x)$ is a df.

Here, the aim is to characterize a family of distributions defined in (3) and (4) by considering conditional expectation of functions of lower record values when the conditioning is on any record value, not necessarily the adjacent one. Various well-known distributions (e.g., Power Function, Pareto, Inverse Weibull, Cauchy) arise from the above family of distributions by suitable choices of $\mathrm{h}(x)$ and the constants $a, b$ and $c$.

\section{Characterization Theorems}

Theorem 1: $\quad$ Let $X$ be an absolutely continuous random variable with df $\mathrm{F}(x)$ and $\operatorname{pdf} \mathrm{f}(x)$ on support $(\alpha, \beta)$, where $\alpha$ and $\beta$ may be finite or infinite. Then, for $1 \leq j<s$,

$$
\mathrm{E}\left[\mathrm{h}\left(X_{\mathrm{L}(s)}\right) \mid X_{\mathrm{L}(j)}=x\right]=a_{s \mid j} \mathrm{~h}(\mathrm{x})+\mathrm{b}_{s \mid j}, j=r, r+1
$$

if and only if 


\section{CHARACTERIZATIONS OF DISTRIBUTIONS}

$$
\mathrm{F}(x)=[a \mathrm{~h}(x)+b]^{c}, x \in(\alpha, \beta)
$$

where

$$
a_{s \mid j}=\left(\frac{c}{c+1}\right)^{s-j}, \quad b_{s \mid j}=-\frac{b}{a}\left(1-a_{s \mid j}\right)
$$

and $\mathrm{h}(x)$ is a monotonic and differentiable function of $x$ such that $\mathrm{h}(x) \rightarrow 0$ as $x \rightarrow \beta$ and $\mathrm{h}(x) \mathrm{F}(x) \rightarrow 0$ as $x \rightarrow \alpha$.

Proof: $\quad$ First, prove (6) implies (5):

$$
\mathrm{F}(x)=[a \mathrm{~h}(x)+b]^{c}, \mathrm{f}(x)=a c \mathrm{~h}^{\prime}(x)[a \mathrm{~h}(x)+b]^{c-1}
$$

From (1) and (5),

$$
\begin{aligned}
& \mathrm{E}\left[\mathrm{h}\left(X_{\mathrm{L}(s)}\right) \mid X_{\mathrm{L}(r)}=x\right] \\
& =\frac{1}{\Gamma(s-r)} \int_{\alpha}^{x} \mathrm{~h}(y)[-\ln \mathrm{F}(y)+\ln \mathrm{F}(x)]^{s-r-1} \frac{\mathrm{f}(y)}{\mathrm{F}(x)} d y \\
& =\frac{1}{\Gamma(s-r)[a \mathrm{~h}(x)+b]^{c}} \int_{\alpha}^{x} \mathrm{~h}(y)\left[c \ln \left(\frac{a \mathrm{~h}(\mathrm{x})+b}{a \mathrm{~h}(\mathrm{y})+b}\right)\right]^{s-r-1} \\
& \times a c \mathrm{~h}^{\prime}(\mathrm{y})[a \mathrm{~h}(y)+b]^{c-1} d y
\end{aligned}
$$

Let

$$
t=\ln \left(\frac{a \mathrm{~h}(x)+b}{a \mathrm{~h}(y)+b}\right)^{c}
$$

then (7) is

$$
\mathrm{E}\left[h\left(X_{\mathrm{L}(s)}\right) \mid X_{\mathrm{L}(r)}=x\right]=\frac{1}{a \Gamma(s-r)} \int_{0}^{\infty}\left[(a \mathrm{~h}(x)+b) \mathrm{e}^{-t / c}-b\right] t^{s-r-1} \mathrm{e}^{-t} d t
$$




\section{FAIZAN ET AL}

which reduces to

$$
\mathrm{E}\left[\mathrm{h}\left(X_{\mathrm{L}(s)}\right) \mid X_{\mathrm{L}(r)}=x\right]=\left(\frac{c}{c+1}\right)^{s-r} \mathrm{~h}(x)+\frac{b}{a}\left[\left(\frac{c}{c+1}\right)^{s-r}-1\right]
$$

and hence the 'if' part.

To prove (5) implies (6),

$$
\frac{1}{\Gamma(s-r)} \int_{\alpha}^{x} \mathrm{~h}(y)[-\ln \mathrm{F}(y)+\ln \mathrm{F}(x)]^{s-r-1} \frac{\mathrm{f}(y)}{\mathrm{F}(x)} d y=a_{s \mid r} \mathrm{~h}(x)+b_{s \mid r}=\mathrm{g}_{s \mid r}(x)
$$

or

$$
\frac{1}{\Gamma(s-r)} \int_{\alpha}^{x} \mathrm{~h}(y)[-\ln \mathrm{F}(y)+\ln \mathrm{F}(x)]^{s-r-1} \mathrm{f}(y) d y=\mathrm{g}_{s \mid r}(x) \mathrm{F}(x)
$$

Differentiating both the sides of (8) with respect to $x$ and re-arranging the terms,

$$
\frac{\mathrm{f}(x)}{\mathrm{F}(x)}=\frac{\mathrm{g}_{s \mid r}^{\prime}(x)}{g_{s \mid r+1}(x)-\mathrm{g}_{s \mid r}(x)}=\mathrm{A}(x)
$$

Now

$$
\mathrm{g}_{s \mid r+1}(x)-\mathrm{g}_{s \mid r}(x)=\frac{\left(\frac{c}{c+1}\right)^{s-r-1}[a \mathrm{~h}(x)+b]}{a(c+1)}
$$

Therefore

$$
\mathrm{A}(x)=\frac{a c \mathrm{~h}^{\prime}(x)}{[a \mathrm{~h}(x)+b]}
$$

and hence 


\section{CHARACTERIZATIONS OF DISTRIBUTIONS}

$$
\frac{\mathrm{f}(x)}{\mathrm{F}(x)}=\frac{a c \mathrm{~h}^{\prime}(x)}{[a \mathrm{~h}(x)+b]}
$$

Thus

$$
\mathrm{F}(x)=[a \mathrm{~h}(x)+b]^{c}
$$

and hence the sufficiency part.

Theorem 2: Under the conditions given in the Theorem 1 and for $1 \leq j \leq r<s$,

$$
\mathrm{E}\left[\mathrm{h}\left(X_{\mathrm{L}(s)}\right) \mid X_{\mathrm{L}(j)}=x\right]=a_{s \mid j} \mathrm{E}\left[\mathrm{h}\left(X_{\mathrm{L}(r)}\right) \mid X_{\mathrm{L}(j)}=x\right]+b_{s \mid j}, j=m, m+1
$$

if and only if (6) holds, where $a_{s \mid j}$ and $b_{s \mid j}$ are defined as in Theorem 1.

Proof: $\quad$ In the view of Theorem 1,

$$
\mathrm{E}\left[\mathrm{h}\left(X_{\mathrm{L}(s)}\right) \mid X_{\mathrm{L}(m)}=x\right]=a_{s \mid m} \mathrm{~h}(x)+b_{s \mid m}
$$

and

$$
\mathrm{E}\left[\mathrm{h}\left(X_{\mathrm{L}(r)}\right) \mid X_{\mathrm{L}(m)}=x\right]=a_{r \mid m} \mathrm{~h}(x)+b_{r \mid m}
$$

Now,

$$
\begin{aligned}
a_{s \mid m} & =\left(\frac{c}{c+1}\right)^{s-m} \\
& =\left(\frac{c}{c+1}\right)^{s-r}\left(\frac{c}{c+1}\right)^{r-m}=a_{s \mid r} a_{r \mid m}
\end{aligned}
$$

Therefore 


$$
\begin{aligned}
\mathrm{E}\left[\mathrm{h}\left(X_{\mathrm{L}(s)}\right) \mid X_{\mathrm{L}(m)}=x\right] & =a_{s \mid m} \mathrm{~h}(x)+b_{s \mid m} \\
& =a_{s \mid r} a_{r \mid m} \mathrm{~h}(x)+b_{s \mid m} \\
& =a_{s \mid r}\left[a_{r \mid m} \mathrm{~h}(x)+b_{r \mid m}\right]+b_{s \mid r} \\
& =a_{s \mid r} \mathrm{E}\left[\mathrm{h}\left(X_{\mathrm{L}(r)}\right) \mid X_{\mathrm{L}(m)}=x\right]+b_{s \mid r}
\end{aligned}
$$

and hence the necessary part.

For the sufficiency part,

$$
\begin{aligned}
& \frac{1}{\Gamma(s-m)} \int_{a}^{x} \mathrm{~h}(y)[-\ln \mathrm{F}(y)+\ln \mathrm{F}(x)]^{s-m-1} \mathrm{f}(y) d y \\
& \quad=a_{s \mid r} \frac{1}{\Gamma(r-m)} \int_{\alpha}^{x} \mathrm{~h}(y)[-\ln \mathrm{F}(y)+\ln \mathrm{F}(x)]^{r-m-1} \mathrm{f}(y) d y+b_{s \mid r} \mathrm{~F}(x)
\end{aligned}
$$

Differentiating both the sides of (14) with respect to $x$,

$$
\begin{aligned}
\frac{1}{\Gamma(s-r)} \int_{\alpha}^{x} \mathrm{~h}(y)[-\ln \mathrm{F}(y)+\ln \mathrm{F}(x)]^{s-r-1} \frac{\mathrm{f}(y)}{\mathrm{F}(x)} d y & =a_{s \mid r} \mathrm{~h}(x)+b_{s \mid r} \\
& =\mathrm{g}_{s \mid r}(x)
\end{aligned}
$$

Proceeding as in Theorem 1 gives the result.

Table 1. Examples based on the df $\mathrm{F}(x)=[a h(x)+b]^{c}$

\begin{tabular}{rrrrrr} 
Distribution & $\mathbf{F}(\boldsymbol{x})$ & $\boldsymbol{a}$ & $\boldsymbol{b}$ & $\boldsymbol{c}$ & $\mathbf{h}(\boldsymbol{x})$ \\
\hline Power function & $a^{-p} x^{p}, 0<x \leq a$ & $a^{-q}$ & 0 & $p / q$ & $x^{q}$ \\
Pareto & $1-a^{p} X^{p}, a \leq x<\infty$ & $a^{-1}$ & 0 & $p$ & $x$ \\
Inverse Weibull & $\mathrm{e}^{-\theta x^{p}}, 0 \leq x<\infty$ & 1 & 0 & 1 & $x^{p}, p>0$ \\
& & 1 & 0 & $\theta$ & $e^{-\theta x^{p}}$ \\
Burr type III & {$\left[1+\theta x^{p}\right]^{-\lambda}, 0 \leq x<\infty$} & $\theta$ & 1 & $-\lambda$ & $x^{-x^{p}}$ \\
& 1 & 1 & 1 & $-\lambda$ & $\theta x^{p}, p, \lambda \neq 1$ \\
Cauchy & $\frac{1}{2}+\frac{1}{\pi} \tan ^{-1}\left(\frac{x-\theta}{\lambda}\right)$, & $\frac{1}{\pi}$ & $\frac{1}{2}$ & 1 & $\tan ^{-1}\left(\frac{x-\theta}{\lambda}\right)$, \\
& $-\infty<x<\infty$ & & & & $-\infty<\theta<\infty, \lambda>0$ \\
\hline
\end{tabular}




\section{CHARACTERIZATIONS OF DISTRIBUTIONS}

Remark 1: $\quad$ At $r=j$, Theorem 2 reduces to Theorem 1 .

Remark 2: At $a=-a / c, b=1$, and $c \rightarrow \infty, \mathrm{F}(x)=[a \mathrm{~h}(x)+b]^{c} \rightarrow \mathrm{e}^{-a \mathrm{~h}(x)}$ as obtained by Faizan and Khan (2011).

Theorem 3: $\quad$ Let $X$ be an absolutely continuous random variable with df $\mathrm{F}(x)$ and $\operatorname{pdf} \mathrm{f}(x)$ on support $(\alpha, \beta)$, where $\alpha$ and $\beta$ may be finite or infinite. Then, for $r \leq s<t$,

$$
\mathrm{E}\left[\mathrm{h}\left(X_{\mathrm{L}(s)}\right)-\mathrm{h}\left(X_{\mathrm{L}(r)}\right) \mid X_{\mathrm{L}(t)}=\mathrm{y}\right]=-\frac{1}{a} \sum_{j=r}^{s-1} \frac{1}{j}
$$

if and only if

$$
\mathrm{F}(x)=\exp \left[-\mathrm{e}^{-a \mathrm{~h}(x)+b}\right], x \in(\alpha, \beta)
$$

Proof: $\quad$ In view of (17), it follows

$$
\begin{aligned}
& \mathrm{E}\left[\mathrm{h}\left(X_{\mathrm{L}(s)}\right) \mid X_{\mathrm{L}(t)}=y\right] \\
& =\frac{\Gamma(t)}{\Gamma(s) \Gamma(t-s)} \int_{y}^{\beta} \mathrm{h}(x) \frac{\left[-\ln \mathrm{e}^{-\mathrm{e}^{-a \mathrm{~h}(x)+b}}\right]^{s-1}}{\left[-\ln \mathrm{e}^{-\mathrm{e}^{-a \mathrm{~h}(y)+b}}\right]^{s}}\left[1-\frac{\ln \mathrm{e}^{-\mathrm{e}^{-a \mathrm{~h}(x)+b}}}{\ln \mathrm{e}^{-\mathrm{e}^{-a \mathrm{~h}(y)+b}}}\right]^{t-s-1} a \mathrm{~h}^{\prime}(x) \mathrm{e}^{-a \mathrm{~h}(y)+b} d x \\
& =\frac{\Gamma(t)}{\Gamma(s) \Gamma(t-s)} \int_{y}^{\beta} \mathrm{h}(x)\left[\frac{\mathrm{e}^{-a \mathrm{~h}(x)+b}}{\mathrm{e}^{-a \mathrm{~h}(y)+b}}\right]^{s-1}\left[1-\frac{\mathrm{e}^{-a \mathrm{~h}(x)+b}}{\mathrm{e}^{-a \mathrm{~h}(y)+b}}\right]^{t-s-1} \frac{a \mathrm{~h}^{\prime}(x) \mathrm{e}^{-a \mathrm{~h}(y)+b}}{\mathrm{e}^{-a \mathrm{~h}(y)+b}} d x
\end{aligned}
$$

Setting

$$
u=\frac{\mathrm{e}^{-a \mathrm{~h}(x)+b}}{\mathrm{e}^{-a \mathrm{~h}(y)+b}}
$$

obtains

$$
\mathrm{E}\left[\mathrm{h}\left(X_{\mathrm{L}(s)}\right) \mid X_{\mathrm{L}(t)}=y\right]=\frac{\Gamma(t)}{\Gamma(s) \Gamma(t-s)} \int_{0}^{1}\left(\mathrm{~h}(y)-\frac{1}{a} \ln u\right) u^{s-1}(1-u)^{t-s-1} d u
$$




\section{FAIZAN ET AL}

or

$$
\mathrm{E}\left[\mathrm{h}\left(X_{\mathrm{L}(s)}\right) \mid X_{\mathrm{L}(t)}=y\right]=\mathrm{h}(y)-\frac{1}{a} \frac{\Gamma(t)}{\Gamma(s) \Gamma(t-s)} \int_{0}^{1} \frac{1}{a} \ln u u^{s-1}(1-u)^{t-s-1} d u
$$

From Gradshteyn and Ryzhik (2007, p. 540)

$$
\int_{0}^{1} x^{u-1}\left(1-x^{r}\right)^{v-1} \ln x d x=\frac{1}{r^{2}} \mathrm{~B}\left(\frac{u}{r}, v\right)\left[\psi\left(\frac{u}{r}\right)-\psi\left(\frac{u}{r}+v\right)\right]
$$

where

$$
\begin{gathered}
\mathrm{B}(a, b)=\frac{\Gamma(a) \Gamma(b)}{\Gamma(a+b)}, \quad \psi(a)=\frac{d}{d a} \ln \Gamma(a) \\
\frac{\Gamma(t)}{\Gamma(s) \Gamma(t-s)} \int_{0}^{1} \ln u u^{s-1}(1-u)^{t-s-1} d u=\psi(s)-\psi(t)
\end{gathered}
$$

Therefore

$$
\mathrm{E}\left[\mathrm{h}\left(X_{\mathrm{L}(s)}\right) \mid X_{\mathrm{L}(t)}=y\right]=\mathrm{h}(y)-\frac{1}{a}[\psi(s)-\psi(t)]
$$

Thus

$$
\mathrm{E}\left(\mathrm{h}\left(X_{\mathrm{L}(s)}\right)-\mathrm{h}\left(X_{\mathrm{L}(r)}\right) \mid X_{\mathrm{L}(t)}=y\right)=-\frac{1}{a}[\psi(s)-\psi(r)]
$$

Using the result (Medina \& Moll, 2009), for $n \in \mathbb{N}$,

$$
\psi(n)=-\gamma+\sum_{k=1}^{n-1} \frac{1}{k}
$$

Therefore, 


\section{CHARACTERIZATIONS OF DISTRIBUTIONS}

$$
\mathrm{E}\left(\mathrm{h}\left(X_{\mathrm{L}(s)}\right)-\mathrm{h}\left(X_{\mathrm{L}(r)}\right) \mid X_{\mathrm{L}(t)}=y\right)=-\frac{1}{a} \sum_{j=r}^{s-1} \frac{1}{j}
$$

which proves the necessary part.

For the sufficiency part, let

$$
\begin{gathered}
k=\frac{1}{a} \sum_{j=r}^{s-1} \frac{1}{j} \\
\frac{\Gamma(t)}{\Gamma(s) \Gamma(t-s)} \int_{y}^{\beta} \mathrm{h}(x) \frac{[-\ln \mathrm{F}(x)]^{s-1}}{[-\ln \mathrm{F}(y)]^{t-1}}[-\ln \mathrm{F}(y)+\ln \mathrm{F}(x)]^{t-s-1} \frac{\mathrm{f}(x)}{\mathrm{F}(x)} d x \\
-\frac{\Gamma(t)}{\Gamma(r) \Gamma(t-r)} \int_{y}^{\beta} \mathrm{h}(x) \frac{[-\ln \mathrm{F}(x)]^{r-1}}{[-\ln \mathrm{F}(y)]^{t-1}}[-\ln \mathrm{F}(y)+\ln \mathrm{F}(x)]^{t-r-1} \frac{\mathrm{f}(x)}{\mathrm{F}(x)} d x \\
=-k
\end{gathered}
$$

Differentiating both sides of (19) with respect to $y$,

$$
\begin{aligned}
& \frac{\Gamma(s)}{\Gamma(r) \Gamma(s-r)} \int_{y}^{\beta} \mathrm{h}(x) \frac{[-\ln \mathrm{F}(x)]^{r-1}}{[-\ln \mathrm{F}(y)]^{s-1}}[-\ln \mathrm{F}(y)+\ln \mathrm{F}(x)]^{s-r-1} \frac{\mathrm{f}(x)}{\mathrm{F}(x)} d x \\
& =\mathrm{h}(y)+k=\mathrm{g}_{r \mid s}(y)
\end{aligned}
$$

Again, differentiating (20) with respect to $y$ and simplifying,

$$
-\frac{\mathrm{f}(y)}{\mathrm{F}(y) \ln \mathrm{F}(y)}=\frac{\mathrm{g}_{r \mid s}^{\prime}(y)}{\left[(s-1) \mathrm{g}_{r \mid s-1}(y)-\mathrm{g}_{r \mid s}(y)\right]}=\mathrm{A}(y)
$$

Using the result (Khan, Anwar, \& Chisti, 2010)

$$
\mathrm{F}(x)=\exp \left[-\mathrm{e}^{-\int_{p}^{*} \mathrm{~A}(t) d t}\right]
$$


where $-\ln \mathrm{F}(p)=1$. Thus $\mathrm{F}(x)=\exp \left[-\mathrm{e}^{-a \mathrm{~h}(x)+b}\right]$ and hence the theorem.

\section{Examples}

Proper choice of $a, b$, and $\mathrm{h}(x)$ characterize the distributions as given below:

\section{(i) Power Function Distribution}

$a=1, b=\ln p, \mathrm{~h}(x)=-\ln (-\ln (x / a))$

$\mathrm{F}(x)=(x / a)^{p}, 0<x<a$

\section{(ii) Inverse Weibull Distribution}

$a=1, b=\ln \theta, \mathrm{h}(x)=-\ln x^{-p}$

$\mathrm{F}(x)=\exp \left(-\theta x^{-p}\right), 0<x<\infty$

(iii) Gumbel Distribution

$a=1, b=0, \mathrm{~h}(x)=x$

$\mathrm{F}(x)=\exp \left(-\mathrm{e}^{-x}\right),-\infty<x<\infty$

\section{(iv) Extreme Value-II Distribution}

$a=1, b=\ln \theta^{p}, \mathrm{~h}(x)=\ln x^{p}$

$\mathrm{F}(x)=\exp (-\theta / x)^{p},-\infty<x<\infty$

\section{(v) Logistic Distribution}

$a=1, b=0, \mathrm{~h}(x)=-\ln \left(\ln \left(1+\mathrm{e}^{-c}\right)\right)$

$\mathrm{F}(x)=\left(1+x^{-c}\right)^{-k},-\infty<x<\infty$

\section{(vi) Burr Type-II Distribution}

$a=1, b=\theta, \mathrm{h}(x)=-\ln \left(\ln \left(1+\mathrm{e}^{-x}\right)\right)$

$\mathrm{F}(x)=\left(1+\mathrm{e}^{-x}\right)^{-\theta},-\infty<x<\infty$

\section{(vii) Burr Type-III Distribution}

$a=1, b=\ln k, \mathrm{~h}(x)=-\ln \left(\ln \left(1+x^{-c}\right)\right)$

$\mathrm{F}(x)=\left(1+x^{-c}\right)^{-k}, 0<x<\infty$ 


\section{CHARACTERIZATIONS OF DISTRIBUTIONS}

\section{(viii) Burr Type-IV Distribution}

$$
\begin{aligned}
& a=1, b=\ln k, \mathrm{~h}(x)=-\ln \left(\ln \left(1+\left(\frac{c-x}{x}\right)^{\frac{1}{c}}\right)\right) \\
& \mathrm{F}(x)=\left(1+\left(\frac{c-x}{x}\right)^{\frac{1}{c}}\right)^{-k}, 0<x<c
\end{aligned}
$$

\section{(ix) Burr Type-V Distribution}

$a=1, b=\ln k, \mathrm{~h}(x)=-\ln \left(\ln \left(1+c \mathrm{e}^{-\tan x}\right)\right)$

$\mathrm{F}(x)=\left(1+c \mathrm{e}^{-\tan x}\right)^{-k},-\pi / 2<x<\pi / 2$

\section{(x) Burr Type-VI Distribution}

$a=1, b=\ln k, \mathrm{~h}(x)=-\ln \left(\ln \left(1+c \mathrm{e}^{-k \sinh x}\right)\right)$

$\mathrm{F}(x)=\left(1+c \mathrm{e}^{-k \sinh x}\right)^{-k},-\infty<x<\infty$

\section{Acknowledgements}

The authors are thankful to the anonymous Reviewer and Editor for their comments and suggestions, which resulted in an improvement in the presentation of this manuscript.

\section{References}

Ahsanullah, M. (1995). Record statistics. New York, NY: Nova Science Publishers.

Ahsanullah, M., Shakil, M., \& Golam Kibria, B. M. (2013). A characterization of power function distribution based on lower record values. ProbStat Forum, 6, 68-72. Retrieved from http://probstat.org.in/PSF-2013-08.pdf Arnold, B. C., Balakrishnan, N., \& Nagaraja, H. N. (1998). Records. New York, NY: Wiley. doi: 10.1002/9781118150412

Azedine, G. (2013). Characterization of the power distribution based on the lower records. Applied Mathematical Sciences, 7(106), 5259-5267. doi: 


\section{FAIZAN ET AL}

Faizan, M., \& Khan, M. I. (2011). A characterization of continuous distributions through lower record statistics. ProbStat Forum, 4, 39-43.

Gradshteyn, I. S., \& Ryzhik, I. M. (2007). Table of integrals, series, and products (7th ed.). Amsterdam: Academic Press.

Khan, A. H., Anwar, Z., \& Chisti, S. (2010). Characterization of continuous distributions through conditional expectation of functions of dual generalized order statistics. Pakistan Journal of Statistics, 26(4), 615-628.

Malinowska, I., \& Szynal, D. (2008). On characterization of certain distribution of $k$ th lower (upper) record values. Applied Mathematics Computation, 202(1), 338-347. doi: 10.1016/j.amc.2008.02.022

Medina, L. A., \& Moll, V. H. (2009). The integrals in Gradshteyn and Ryzhik. Part 10: The digamma function. SCIENTIA. Series A: Mathematical Sciences, 17, 45-66. Retrieved from http://www.mat.utfsm.cl/scientia/archivos/vol17/ar6.pdf

Nadarajah, S., Teimouri, M., \& Shih, S. H. (2014). Characterizations of the Weibull and uniform distributions using record values. Brazilian Journal of Probability and Statistics, 28(2), 209-222. doi: 10.1214/12-bjps202

Nevzorov, V. B. (2001). Records: Mathematical theory. Providence, RI: American Mathematical Society.

Shawki, A. I., \& Bakoban, R. A. (2009). Conditional expectation of certain distributions of record values. International Journal of Mathematical Analysis, 3(17), 829-838. Retrieved from http://www.m-hikari.com/ijma/ijma-password2009/ijma-password17-20-2009/bakobanIJMA17-20-2009.pdf

Yanev, G. P. (2012). Characterization of exponential distribution via regression of one record value on two non-adjacent record values. Metrika, 75(6), 743-760. doi: 10.1007/s00184-011-0350-z 